\title{
Challenges Faced in the Integration of Pharmacogenetics/Genomics into Drug Development
}

Brandi Maria Luisa ${ }^{1 *}$, Abadie Eric ${ }^{2}$, Daly Ann ${ }^{3}$, Dere Willard ${ }^{4}$, Ethgen Dominique ${ }^{5}$, Goel Niti ${ }^{6}$, Gouze Jean-Noel ${ }^{7}$, Ingelman-Sundberg Magnus ${ }^{8}$, Kaufman Jean-Marc ${ }^{9}$, Laslop Andrea ${ }^{10}$, Laurie David ${ }^{11}$, Maliepaard Marc ${ }^{12}$, McHale Duncan ${ }^{13}$, Meyer Joanne ${ }^{14}$, Mitlak Bruce ${ }^{15}$, Paulmichel Markus $^{16}$, Pirmohamed Munir ${ }^{17}$, Reginster Jean-Yves ${ }^{18}$, Rizzoli René ${ }^{19}$, Tsouderos Yannis ${ }^{20}$, Vonderscher Jacky ${ }^{21}$ and Flamion Bruno ${ }^{22}$ on behalf of the Group for the Respect of Ethics and Excellence in Science (GREES)

${ }^{1}$ Department of Internal Medicine, University of Florence, Florence, Italy

${ }^{2}$ Direction Générale, Afssaps 143, 147, Bld Anatole, Saint Denis, France

${ }^{3}$ Newcastle University Medical School, Framlington Place, Newcastle upon Tyne NE24HH, UK

${ }^{4}$ Amgen Limited, 1 Uxbridge, UK Business Part, Sanderson Road, UB8 1DH, UK

${ }^{5}$ Medimmune, One Medimmune Way Gaithersburg, MD 20878, USA

${ }^{6}$ UCB 1950 Lake Park Drive Smyrna, GA 30080, USA

${ }^{7}$ Laboratoires Genevrier s.a., 280, Rue de GOA, Z.I. Les Trois Moulins 06600 Antibes, France

${ }^{8}$ Karolinska Institute of environmental Medecine, SE-171 77 Stockholm Nobels väg 5, Solna, Sweden

${ }^{9}$ Ghent University Hospital-De Pintelaan 185 B-9000 Gent, Belgium

${ }^{10}$ A GES PharmMed, Institute for Science and Information, Schnirchgasse 9, 1030 Vienna, Austria

${ }^{11}$ Novartis Pharma AG, Regulatory Affairs, Novartis Campus, St Johann, 4002 Basel, Switzerland

${ }_{12}^{12}$ utch Medicines Agency, 579 CBG/MEB - University Medical Centre St Radboud, PO Box 9101, 6500 HB Nijmegen, The Netherland

${ }^{13}$ ASTRAZENECA-Translational Sciences - DECS Mereside, 8F29, Alderley Park, SK10 4TG Macclesfield - Cheshire UK

${ }^{14}$ Novartis Institutes for BioMedical Research - BioMarker Development, 220 Massachussets Ave- 02139 Cambridge - MA, USA

${ }^{15}$ Eli Lilly \& Co, Lilly corporate center, D.C. 6134, Indianapolis, IN 46285, USA

${ }^{16}$ Paracelsus Medical University, Pharmacology and toxicology, Strubergasse 21, 5020 Salzburg, Austria

${ }_{17}$ University of Liverpool - Pharmacology, Sherrington Building Ashton Street - L69 3GE - Liverpool, UK

${ }^{18} \mathrm{CHU}$ Centre Ville, 45 Quai Godefroid Kurth, 4020 Liege, Belgium

${ }^{19}$ University Hospital Bone Diseases Division, 1211 Geneva, Switzerland

${ }^{2}$ Institutions de Recherches Internationales SERVIER, 6, Place des Pléïades, 92400 Courbevoie, France

${ }^{21}$ F.Hoffmann - La Roche LTD Global Molecular Medecine LABS-PDL, Grenzacherstr 124, 4070 Basel, Switzerland

${ }^{22}$ University of Namur FUNDP, Department Phisiology and Pharmacology, 61 rue de Bruxelles, 5000 Namur, Belgium

\begin{abstract}
Pharmacogenetics and pharmacogenomics are rapidly developing areas of research, with potentially enormous clinical implications and an increasing number of requests for inclusion of pharmacogenetic discoveries in drug labels. While academic centers have been central to the development of research in this area, the need for collaboration amongst academia, industry and regulators is important in order to proceed with high quality and utmost speed.

As a number of issues need to be addressed jointly by the different parties, the Group for the Respect of Ethics and Excellence in Science, a not-for-profit organization that constitutes an interface between regulators, academic scientists and representative of the pharmaceutical industry in Europe, organized a meeting to discuss the use of pharmacogenetics/genomics in drug development.

The areas for discussion amongst the participants included design of pharmacogenetic studies, regulatory requirements in the European Union, pharmacokinetic and pharmacodynamic aspects with a proposal on future prospects for recognition of pharmacogenetic biomarkers in Europe. A common principle agreed by the group was that there was a need to discover much earlier areas that would have an impact on the safety or efficacy of a new drug. Future guidelines should offer guidance using different scenarios with the common goal of improving the process of drug development.
\end{abstract}

Keywords: Pharmacogenetics; Pharmacogenomics; Regulatory agencies; Industry; Biomarkers

\section{Introduction}

Pharmacogenomics and pharmacogenetics have the potential to improve the discovery, development, and use of medicines. Clinically relevant pharmacogenetic examples, involving variation in single or small number of genes mostly related to drug metabolism have been known for decades. The concept of pharmacogenetics has evolved into pharmacogenomics, with a shift from a focus on individual candidate genes to genome-wide association studies.

Since the first pharmacogenetic discovery of glucose-6-phosphate dehydrogenase deficiency resulting in hemolysis after primaquine treatment [1], the field has been attracting increasing interest with a promise of enhancing healthcare delivery in the $21^{\text {st }}$ century. With major advances in genomic science and its application to drug response, the number of publications associated with pharmacogenomics have been dramatically increased [2].

Genomics has been able to explain interindividual variability in

*Corresponding author: Maria Luisa Brandi, MD, PhD, Department of Interna Medicine, University of Florence, Viale Pieraccini, 650139 Florence, Italy, Tel: +39 055 7946304; Fax: +39 055 7946303; E-mail: m.brandi@dmi.unifi.it

Received February 16, 2012; Accepted March 28, 2012; Published April 30, 2012

Citation: Luisa BM, Eric A, Ann D, Willard D, Dominique E, et al. (2012) Challenges Faced in the Integration of Pharmacogenetics/Genomics into Drug Development. J Pharmacogenom Pharmacoproteomics 3:108. doi:10.4172/2153-0645.1000108

Copyright: ( 2012 Luisa BM, et al. This is an open-access article distributed under the terms of the Creative Commons Attribution License, which permits unrestricted use, distribution, and reproduction in any medium, provided the original author and source are credited. 
pharmacokinetics and pharmacodynamics of medicinal products, also influenced by factors including age, gender, weight, renal, and hepatic function. Markers of genomic variation included single nucleotide polymorphisms (SNPs), DNA insertions/deletions, and gene copy number variation (CNV).Certainly, the actual and future sequencing techniques will facilitate rapid whole-genome sequencing. Moreover, the establishment of large biobanks from well-characterized patients, also in collaboration with the pharmaceutical industry, will offer novel and unique opportunities. Impact of environmental factors on individual variability to drug response should also be evaluated through genomic science (Figure 1). Recently, the epigenome has attracted great interest in pharmacogenomic response, providing a foundation for future studies exploring key epigenetic modifications (i.e., differences in phenotype/gene expression caused by mechanisms other than differences in the underlying DNA sequence) [3]. Moreover, control of gene expression by microRNAs and alternative splicing are cellular mechanisms that through influence on proteome diversity can affect variability in drug response $[4,5]$. The future integration of genomics, proteomics, metabolomics, and epigenetics will likely facilitate identification of genetic factors associated with drug response.

Theoretically, genomic biomarkers should offer the advantage of improved specificity and reduction of heterogeneity, becoming very attractive in drug development, for the potential ability to reduce overall developmental costs and to use novel development strategies at various stages, both in pre-clinical and clinical phases. The environment of innovation in pharmacogenomic knowledge, combined with the development of advanced genetic technologies, has affected the number of requests for inclusion of pharmacogenomic discoveries in drug labelling [6,7]. Pharmacogenomic principles are now being used in drug development to, for example, identify the best dose for all carriers, without this becoming necessarily manifested in drug labels. The availability of the therapeutic dose recommendations during the process of therapeutic decision making represents already an important step in the clinical use of pharmacogenetic information.

As both genomic science and its application to drug response were undergoing major advances [8,9], a number of stakeholders not always acted in a coordinated way. The regulatory agencies, both the United States (US) Food and Drug Administration (FDA) and the European Medicines Agency (EMA), recognized pharmacogenetics as an opportunity to individualized therapy, raising the issue of the

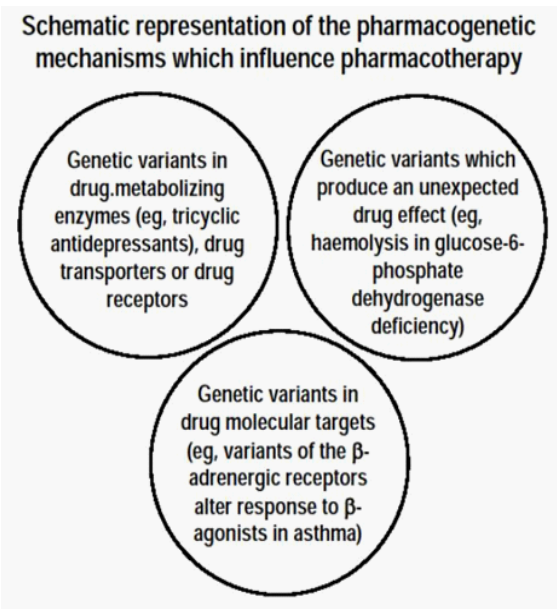

Figure 1: level of evidence required to show clinical utility [10-17]. Academic and professional societies have been central to the development of pharmacogenomic research, focusing mostly on well established drugs and usually publicly founded (Table 1). In parallel, the Industry Pharmacogenomics Working Group (http://i-pwg.org) has published practical perspectives regarding potential guidelines for pharmacogenomic study design and analyses [18].

Altogether these efforts have made it possible to advance the field with significant progress already made, but a number of issues need still to be solved. Certainly, the respective roles of the different stakeholders in the evaluation of pharmacogenomic analyses for the clinic have to be defined. Also, the evaluation of genomic biomarkers in the qualification process in the context of clinical development and assessment of benefit (i.e., risk balance of medicinal products or selection of the relevant target population) should be clearly defined. Moreover, development of genomic biomarkers may involve the construction of specific tests (companion diagnostics) or specific kits (platforms) to detect their presence. No matter what level of evidence is required for each situation, it will be necessary to develop simple clinical algorithms to aid physicians in their interpretation and use of genetic data. Finally, developing the right study designs might help to close the gap between the extensive academic research data on the importance of pharmacogenomics in medicine and the very limited introduction of this knowledge in clinical practice.

For all these reasons, the need for a collaboration among regulatory authorities, academia, industry, and other relevant stakeholders in the appropriate application of pharmacogenomic biomarkers is required (Figure 2). The Group for the Respect of Ethics and Excellence in Science (GREES), an non-for-profit organization frequently involved in producing reflection papers in various areas of medicine and ethics and that constitutes an interface between regulators, academic scientists and representatives of the pharmaceutical industry, organized a meeting to provide an overview on evidence-based considerations on pharmacogenomic biomarkers from regulatory and industrial viewpoints. Indeed, while a number of scientific aspects are discussed in several publications, specific issues related to drug development and discussion on regulatory considerations have lagged behind.

The workshop provided the opportunity for a constructive and fruitful dialogue among all stakeholders. The invited participants, all scientists with a recognized knowledge in the field of pharmacogenomics, were supposed to actively participate in the presentations and discussions. Awareness was raised among all attendees regarding the type of challenges that exist in clinical trial decision-making and the substantial unknowns and uncertainties that are integral to drug development, the theme of a soon coming Reflection Paper from the EMA, focused on evidence based considerations of genomic biomarkers in drug development.. The intent of the workshop was, therefore, to help steer the content of this official Reflection Paper through an ample discussion among experts from academia, regulatory bodies, and industry. The meeting and its publication, co-authored by the active participants, provide a summary of those independent discussions.

\section{Current and Future Methodologies to Pharmacogenetic/ Genomic Study Designs}

\section{State of the art}

Genetic polymorphisms may affect all aspects of drug therapy. The influence of genetic variation on drug transport and metabolism is well recognized. Polymorphisms in genes coding for drugs' receptors 


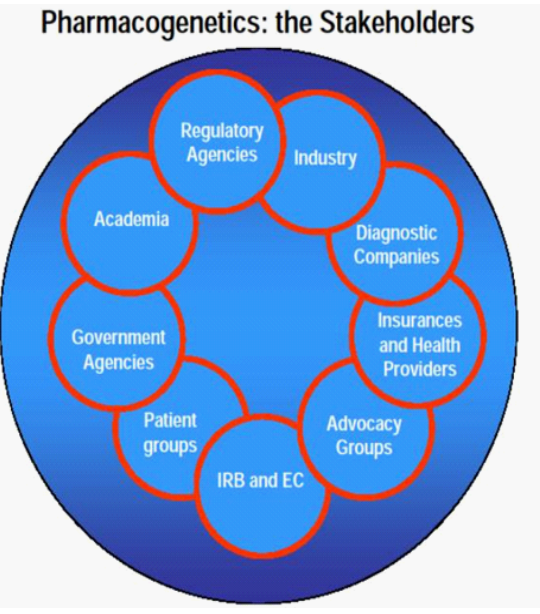

Figure 2:

and signalling pathways (pharmacodynamics) may affect the action of medicines. With regard to pharmacokinetics, polymorphisms in genes coding either for membrane transporters or for drug-metabolizing enzymes result in substantial variations in drug's bioavailability, delivery to the target site, and plasma concentration (pharmacokinetics). Moreover, genetic polymorphisms also affect dose-related adverse drug reactions. However, with so much knowledge accumulated, very few current drugs are selected or dosed in accordance with individual pharmacogenetic biomarkers $[19,20]$.

The reason for the slow pace of the application of genomics to clinical medicine can be found in the challenges associated with the lack of replication of study findings, as the knowledge of clinical pharmacogenomic research methodology was initially poor [21]. Today, both the European and US regulatory agencies and international organizations or boards have issued Guidelines or reports on this subject [22-24].

The two stakeholders on charge for pharmacogenetic/genomic research, the academia and the industry, have been working in parallel, with rare points for common projects to be carried out. On one side, for early drug development the industry concentrates its efforts around pharmacokinetics in terms of mean dose-related exposure, focusing on pharmacogenetic studies in later developmental stages only in the case of drugs with a narrow therapeutic windows. Moreover, the industry tends to focus on genes whose enzymatic consequences are understood (i.e., CYP genes), with a reluctancy to introduce untargeted pharmacogenetics in drug development. On the other side, academic studies, usually publicly founded, often evaluate well-established drugs with known serious adverse events (Table 1), with a focus on science, novel technologies, and mechanisms, usually identified in small pilot studies. Subsequent validation of these markers is, however, difficult, as samples from large controlled studies are rarely available. The harmonization between industry and academia programs could be found through the mutual understanding of quantified risks and benefits, where pharmacogenetic/genomic designs are developed in a discussion that involves regulatory agencies. The potential benefits, along with the type and quality of evidence gained in different study design are evaluated below.

\section{Future methodologies}

The two most common strategies to test a pharmacogenetic/ genomic question are candidate-gene and genome-wide association (GWA) approaches. Candidate-gene investigations should focus on variations/alterations in genes causing anticipated functional effects on gene expression or function of the gene products, thus resulting in marked alterations in phenotype [25]. An example of this is warfarin, where $C Y P 2 C 9^{*} 2$ and $C Y P 2 C 9^{*} 3$ polymorphisms, known to impact on the functionality of the CYP2C9 enzyme, were selected for evaluation and determined to be associated with warfarin's dose requirements [26-28]. This is an example where the pharmacokinetic component (i.e., CYP2C9 polymorphism) is only one factor influencing dose requirement and is complemented by pharmacodynamic variation in the VKORC1 gene, which codes for the enzyme inhibited by warfarin, which together provides much better assessment of variability. The process needed to select candidate-genes is not always so straightforward, as the functional effects may not be known, and the choice has to be made from several polymorphisms. The selection of SNPs can be based on allele frequency or whether the SNP acts as a "tag" for other SNPs through the process of linkage disequilibrium [29, 30]. It is acknowledged that stratification by genotype is difficult for very rare genetic variants, where individual studies will not have adequate statistical power to detect the effect, and pooled analysis of study data or formal meta-analysis may be valuable. Major disadvantages of the candidate-gene approach are that knowledge of the function of the gene regarding the drug response should be known in advance and that, given the risk of false association, the findings have to be replicated in at least one independent population [31]. However, this approach has the fundamental advantages of being less expensive and of requiring a smaller sample size than the genome-wide type of methodology [32].

With the advance of technology, more global profiling of, for example, inter-individual variability can be provided through the use of the absorption, distribution, metabolism, and excretion (ADME) chip. This method represents a well validated analytical platform which is widely available and encompasses 1,936 drug metabolism markers in 225 gernes. An alternative is the use of genome-wide chips, with the advantage to evaluate variants in both pharmacokinetic and pharmacodynamic pathways. In addition, this approach can make possible the discovery of new pharmacogenomic markers, for example those associated with adverse drug reactions, not always predictably associated with the known pharmacology of a drug [33]. However, these types of approach require large number of patients and are, therefore, costly. Moreover, as with the candidate-gene association design, the results have to be replicated in one independent population [31].

\section{Study designs}

Postmarketing pharmacogenetic/genomic research has been using various study designs for assessing efficacy and safety.

1. Drug-induced Liver Injury

-DILIGEN (UK)

-EUDRAGENE (Europe)

-DILIN (US)

-Spanish DILI Registry

2. Long QT

-DARE (UK)

3. Stain-induced Myopathy

-SEARCH collaborative (UK)

-STAGE (UK)

Table 1: Publicly-Funded Pharmacogenetic Studies On Serious Adverse Reactions. 
Individual pharmacogenetic case reports related to adverse drug reactions represent an important source of information, but uniquely as hypothesis-generating data as it happened in carriers of inherited thiopurine methyltransferase deficiency [34].

Frequently, pharmacogenetic/genomic biomarkers are described in non-randomized cohort or single arm studies, also outside of drug development programmes and often lacking pre-defined biomarkerrelated end points. The study designs used encompass cross-sectional studies, case-control studies and studies in cohorts in their natural settings in the absence of controls [35-38]. Lack of replication in these types of designs remains an important issue, often related to lack of robustness, of proper clinical end points, of careful phenotypic quality controls, and of appropriate statistical considerations [18]. These exploratory studies, mostly carried out postmarketing, are usually based on poorly selected cohorts limited in size, with lack of possibility to establish the predictive value, the sensitivity and the specificity for a pharmacogenetic marker. Any conclusions derived from these types of models must be interpreted with caution, and this is true both for candidate-gene and genome-wide approaches. The main limit are external influences or confounding variables [39]. Bias also may play a considerable role in validation of a pharmacogenomic biomarker, as selection and measurement bias are of great importance.

In order to reduce the confounders and bias, pharmacogenetic/ genomic studies should be carried out within controlled clinical studies in the different phases (I, II, and III), either prospectively or retrospectively, up to studies specifically designed to assess the value of pharmacogenetic diagnostics [24,39-41]. Exploratory investigation of genomic biomarkers through randomized clinical trials is often possible where preliminary information regarding the value of a predictive marker is based on published literature. However, even if in a pharmacogenetic study within a randomized controlled clinical drug trial it is possible to evidence the predictive value for a particular drug effect, these studies may be neither possible nor necessary to carry out for any genomic biomarker. In retrospective analyses of completed controlled clinical trials certain elements are critical, such as the size of the sample, quality of the trial, and a pre-defined analysis plan. Analysis of data from two (or more) high quality independent controlled clinical trials would provide the strongest evidence. Certainly, prospective controlled trials for the identification and even validation of genomic biomarkers would be ideal, but very expensive, time and effort intensive, and often need significant preliminary evidence to demonstrate either association or biological plausibility. A prospective trial should be confirmatory in nature for prospective validation of predictive genomic biomarkers, with various forms of design (i.e., unselected, enriched or targeted, hybrid, and adaptive). When new prospectively designed trials are not feasible to test the predictive ability of a marker, a retrospective validation may become possible. It is evident that for any retrospective validation crucial elements are: preliminary data from one or more high quality prospective controlled clinical trials, availability of genomic biomarkers status from a large number of subjects to avoid selection bias, predefined hypothesis as well as analysis plan, and a statistically compelling association adjusted for multiple testing. Overall, even tough prospective controlled pharmacogenomic studies appear as the gold standard, the value of any design may differ depending on the context and the details of how exactly the studies were designed and conducted.

Irrespective of the type of trials used for exploration, it is important to collect information not only on the predictability of the genomic biomarker, but also on its clinical validity. The latter is a complex interplay of sensitivity and specificity and the penetrance of the genomic marker. The developmental studies should be the basis to indicate the feasibility of further evaluation for a given genomic biomarker.

In contrast to the study design described above, in which the pharmacogenetic analysis is performed after the assessment of the study outcomes, in sequential before-after design pharmacogenetic evaluations are performed during the study. In a study with this beforeafter sequence, patients receive drug therapies first before genotyping and then again after genotyping, with individual cross-over design being relatively easy to perform.

Community intervention design, in which a region or a hospital performing pharmacogenetic analyses are compared to other region(s) and hospital(s) that are not performing such tests [42]. In applying this approach the introduction of individualized medicine into medical practice could be facilitated.

\section{Quality of the assays}

Finally, for newly identified genomic biomarkers development of specific assays/kits might be necessary, parallel to the drug development.

As the above study designs offer limitations in some cases and appear unaffordable in others, the need for innovative designs is urgently felt. The focus on patients with severe adverse reactions or on poor responders could open the door to pharmacogenetics in disorders difficult to be treated, even in second- or third-line therapy. Another approach is to develop studies in genotypically pre-selected populations, as pharmacogenetic diagnostics is particularly valuable for sub-groups, especially in the case of rare genotypes [43].

The concept of replication and confirmation become essential in any clinical development of pharmacogenomic research, as the reproducibility of pharmacogenetic, even of data showing strong association, is not over $60 \%[44,45]$. By definition confirmatory studies should be powedered not only to replicate previous studies, but also populations different than those included in the exploratory studies.

Last, but not the least, economical, ethical, social, and regulatory issues associated with genomic medicine are complex and challenging. An example, of ethical issue comes from the observation that, if strong data out of controlled clinical studies indicate the possibility that pharmacogenetic biomarkers can save life, these cannot be disregarded. In this case, proper evaluation of non randomized pharmacogenetic studies can make possible to ensure safety or efficacy of a given compound.

\section{Future prospects}

In conclusion, any clinically oriented research in pharmacogenetics/ genomics should encompass up to three phases: a) hypothesis generation and definition; b) reproducibility; and c) risk-benefit assessment for pharmacogenetic diagnostics to proof that genotype-based therapy is better than therapy without these inputs. The achievements so far have been limited, as reproducible data for most cases are still not available. Based on limited success, it is recognized that the challenges facing individualized drug therapy should be addressed individually at the different stages in clinical development. As the knowledge is increasing, the collaboration with regulatory bodies will become essential.

\section{Regulatory Requirements in the European Union}

The regulatory impact of pharmacogenetics is still a developing 
area. Regulators (including competent authorities, national departments of health, and regulatory agencies) have a significant role in translating pharmacogenetics into clinical practice. Common questions faced by regulators include the consistency of findings and results, the requirement for confirmation of pharmacogenetic data, the applicability of association studies to the clinical area, and the evaluation of the impact of pharmacogenetic testing in clinical practice. The main role of regulators with respect to available pharmacogenetic data is to interpret them with respect to their consistency and clinical applicability, to match them to legal limitations, and to use them to protect and improve public health. All of this is achievable only through tight and constructive interactions between regulators, academia, and industry. In Europe, this interaction has been promoted by the 2005 constitution of the Pharmacogenomic Working Party (PGWP) of the Committee for Medicinal Products for Human Use (CHMP) of the EMA. The PGWP was set up in 2005 by CHMP to assist on scientific discussions relevant to the implementation of pharmacogenomics in medicines development and the impact on regulatory assessment. The components of the PGWP are scientists of recognized reputation in various fields such us genomics, ethics, medicine, and regulatory issues, all relevant to the new genomic technologies in medicinal products development and assessment. Within this group an informal process of sharing scientific and technical information is facilitated and only successively transferred to applicants and regulators. Via briefing meeting the Working Party gets inputs from applicants on the circumstances and rationale under which pharmacogenetic data are generated. These dialogues may contribute to minimizing the risks of creating obstacles to the use of novel technologies. A short summary of the briefing meetings will form a dedicated section in the PGWP minutes to the CHMP. Issues identified and questions debated during the briefing meetings are the basis for scientific advice and marketing authorization applications and future guidance. This Working Party has its own work plan and interacts with other agencies worldwide, such as the US FDA and the Japanese Pharmaceuticals and Medical Devices Agency (PMDA). This interactive network of regulatory bodies provides the opportunity to discuss recent developments and ethical/legal questions.

Regulatory involvement pushing the use of pharmacogenetic biomarkers in both drug development and clinical practice is evidenced by the public documents generated in the past five years by agencies worldwide [10-16]. However, these documents provide only an initial framework for building policy. They are yet insufficient to address the moral, ethical, and economic implications of the application of genotypic information to the development of personalized therapeutics. Before pharmacogenetics can be routinely applied, multiple issues will need to be addressed by various stakeholders: privacy issues concerning the use of genotype information in multiple studies; informed consent and the need (if any) for genetic counseling; public access to genetic testing for prediction of therapeutic response; sample size and eligibility requirements for association study evaluation; public access to genetic testing for prediction of therapeutic response; and standardization of data across patient populations.

The recognition of these problems made it possible for the EMA to implement a validation process recommended or required to allow transition from exploratory to valid pharmacogenetic biomarkers. There are five major qualification phases: internal qualification, external qualification, clinical trials (phase I and II; checking for safety and efficacy), large clinical trials (phase III), and continuous surveillance. The level of confidence that a candidate biomarker can attain depends on the phase of validation that has been reached. Ideally, biomarkers may reach the point of surrogacy and replace known clinical end points. However, this designation requires agreement with regulatory authorities, as well as long-term data and evidence for safety and efficacy, as clearly the consequences of a flawed surrogate biomarker could be significant and adversely impact on public health.

Another area that needs to be addressed is the involvement of Institutional Review Boards and Ethic Committees in pharmacogenetics research. These organizations will review and approve, disapprove or modify all the submitted research proposals concerning human subjects, submitted by the academic community or by the pharmaceutical industry. As research moves in the direction of genome analyses based on computational methods, it becomes increasingly important for participating members of IRBs and Ethic Committees to possess specific knowledge to properly evaluate the possible implications of a pharmacogenetic study. It might be that in the future, institutionalindependent review boards with such specialized knowledge are created that can be contracted to perform study analyses and provide guidance on study conduct [44].

In this direction, Reflection Papers on the use of pharmacogenetics in the pharmacokinetic evaluation of medicinal products were published by the EMA in collaboration with the CHMP and the PGWP $[16,45,46]$. The objective of the work of the PGWP in briefing meetings is to discuss in an informal setting the technical, scientific and regulatory issues that arise by the inclusion of pharmacogenomics in the development strategy and to assess their potential implications in the regulatory process. The papers aimed to harmonize the development and reporting of pharmacogenetic/pharmacokinetic studies, and to inform on the possible consequences of pharmacogenetic results for drug labeling. Since the drafting of these papers, progress in the field continues. In light of the evolution and broad acceptance of genotyping methods, as well as increased experience in the use of such pharmacogenetic methodologies during drug development, an update was considered necessary to align this progress. A Reflection Paper will soon be available, providing expanded and improved guidance for both the pharmaceutical industry and regulatory authorities regarding the application of pharmacogenetic methodologies in pharmacokinetic studies of medicinal products.

The future implementation of pharmacogenomic labelling will encompass different uses [47]. At present mandatory use is given for few drugs, mostly related to oncology, for which a clear basis for responsiveness has been demonstrated [47]. Today, pharmacogenomic information is contained on about $10 \%$ of drug labels approved by the FDA [48]

Finally, initiatives to implement pharmacogenomic information into clinical practice has been adopted by various organizations, in order to provide guidance on the use of such tests in the clinic $[49,50]$.

\section{General Issues with Pharmacogenetics/Genomics on Pharmacokinetics and Pharmacodynamics}

The preclinical data that predict interindividual variations in the efficacy and side effects of the drugs in humans should encompass analyses either in the drug membrane transport and metabolism (pharmacokinetics) or in the targeted pathways (pharmacodynamics). Indeed, pharmacogenetic variations may be predicted from in vitro and in vivo data, usually available before first in human studies, therefore accelerating clinical drug development. 
The pharmacokinetics of all medicinal products are prone to interindividual variability, with the highest level of genetic heterogeneity registered in drug metabolism. Phase I metabolism of approximately $40 \%$ of drugs used clinically is affected by polymorphic enzymes. The most important of these enzymes are the cytochrome P450 enzymes, such as CYP2C9, CYP2C19 and CYP2D6. With respect to phase II enzymes, the genetic variability of the UDP-glucoronosyltransferases, $\mathrm{N}$-acetyltransferase-2 and some methyltransferases have been shown to influence interindividual variability in drug pharmacokinetics.

ADME gene sequences have long been studied for the presence of mutations or polymorphisms leading to functionally relevant genetic variation. These sequence variations have been consistently associated with pharmacokinetic properties of a broad range of drugs in vitro and in vivo. Whether these variations also correlate with variations in clinical outcomes is a matter of debate, and often not fully investigated. The metabolizing enzymes account for $80 \%$ of drugs which have pharmacogenetic data in their label currently. In recent years, examples have also been published on specific gene polymorphisms in drug transporters, with possible contribution to the efficacy and safety of medicinal products $[51,52]$. Drug transporters can be regarded as completing the phase I and II enzyme-based detoxification system; drug uptake delivers the drug to the detoxification system to facilitate metabolism, whereas drug efflux decreases the load on detoxification enzymes [53,54]. It is anticipated that this area will greatly expand in the near future, as knowledge about the role of drug transporters is rapidly developing.

Whereas pharmacogenetic traits influencing drug disposition are now relatively well identified, the genetic variability of drug targets remains to be explored. Many genetic polymorphisms affect drug response by modulating the functions of proteins that are drug direct response. These polymorphisms happen to occur in genes encoding for drug target protein function, for drug-target interaction, or for both [55-61].

Either for pharmacodynamic or pharmacokinetic studies, basic research will continue to be necessary both to understand the pathogenesis of human disorders (making possible the development of targeted therapies) and to recognize the genes encoding for target or drug disposition genes (making possible to uncover drug response variation). It would be difficult to develop a common model of when pharmacogenetics/genomics of pharmacodynamics or pharmacokinetics should be studied, as this will greatly depend on the information offered for each given drug by basic research and explorative studies.

For pharmacokinetics EMA issued a Reflection Paper [16] that focused on the situations in which should the effect of pharmacogenetics on pharmacokinetics be studied. This happens to be needed when exposure to a substance could affect safety and efficacy of its use. The first step is, therefore, the knowledge of the enzymes and proteins involved in drug metabolism and transport, along with the identification of pharmacologically active metabolites. If human in vitro data suggest major involvement of a protein known to be the subject of functionally important polymorphisms, inclusion of genotyping directed to the candidate gene is warranted in early phase I studies. When the involvement of the polymorphic gene has been verified, in vivo studies of the effects of specific polymorphisms on the pharmacokinetics of the pharmacologically active compounds likely to contribute to clinical efficacy and /or safety are recommended. In case of unforeseen pharmacokinetic observations of potential clinical relevance in phase I or subsequent studies, pharmacogenetics of pharmacokinetics should be performed as early as possible in the drug developmental program. This will make possible to increase the amount of data that will support the recommendations for use in the genetic subpopulation(s). If there is a need for a dose adjustment, the following routes could be applied: dose titration regardless of genotype, dosing based on genotype or phenotype, or optional gene-based dosing.

The standardization of criteria for evaluating genetic tests encompass analytic validity, clinical validity, clinical utility and ethical, legal and social implications. However, these concepts are not always clear in pharmacogenetics. Therefore, efforts should be made to incorporate pharmacogenetic methodology into premarked drug development, continuing postmarket observational and mechanistic studies.

\section{Future Perspectives for Development of Pharmacogenetic Biomarkers in Europe}

Throughout the workshop the focus was on methodological issues associated with pharmacogenomic biomarkers in relation to clinical development. A number of questions, partially raised in previous Reflection Papers from EMA [13-16,45,46],were considered of particular interest by the group.

Which is the appropriate trial design or the right time for data analysis of pharmacogenetic/genomic studies in the drug development process?

Evidence regarding the potential impact of pharmacogenetics on drug development is accumulating at a speed that makes previous Reflection Papers from EMA no longer sufficient. Industry, in attempting to incorporate pharmacogenetics/genomics in drug development, is confronted with several issues, including high start-up costs and the potential limited utility of the pharmacogenomic biomarkers for its compounds. The important issues to limit the resistance from the Industry appear to be the employment of the appropriate trial design or data analysis methodology at the right time in the drug development process. Signals for genomic biomarkers may be generated based on theoretical plausibility or based on an association noted in preliminary studies, and then confirmed empirically during the exploratory phase of development of the genomic biomarkers. The group recognized the diverse exploratory development strategies to be considered, as listed in table 2 . The exploratory development of a genomic biomarker would encompass: prospective and retrospective non-randomized and randomized studies. Following the exploratory development, confirmatory studies should be validated either retrospectively or prospectively.

Studies on pharmamacogenetic of pharmacokinetics have been taken most of the attention by the reaserach, as they follow the path already traced within the early drug development models. However polymorphisms in genes encoding for drug target structures (i.e., receptors, targeted enzymes, intracellular signalling molecules, and
Do Nothing
Collect "Insurance" Samples
Proactively Genotype Retrospectively
Genotype Prospectively and Randomize According to Genotype
Select Patients Based on Genotype (specifically exclude or include polymorphisms)

Table 2: Main Exploratory Pharmacogenetic Strategies In Drug Development. 
structural proteins) will become relevant for dosing purposes. This approach is typically represented by the warfarin story [26-30].

Which is the role of the diagnostic performance of the pharmacogenetic/genomic biomarker?

Irrespective of the type of the exploratory trials used, the pharmacogenetic/genomic biomarker should be evaluated for predictability and clinical validity. Indeed, the developmental studies themselves should aim to indicate whether further validation of the biomarker is feasible. The standards of diagnostic performance should conform to the accepted standards for qualification and validation, as previously indicated by EMA [62]. The diagnostic performance relates essentially to evaluation of sensitivity and specificity of the biomarker, and to the penetrance of the genomic sequence. Moreover, extrapolation to populations different than the one tested would need to be adequately justified.

What are the potential external influences on the evaluation of a pharmacogenetic/genomic marker?

Genomic and non-genomic factors not related to the pharmacogenomics of a drug response can influence the therapeutic effect and the adverse events related to a given compound. An example is offered by drug drug interactions (DDI). Polypharmacy, often common in disorders that are progressive and multimorbid in nature, may substantially contribute to harmful effects in such settings via DDI. Apart from the mode of action of the respective drugs, a substantial proportion of adverse effects could have been caused by the pharmacokinetic and pharmacodynamic pathways of the drugs and their interactions. DDI clinical trials are conducted frequently as part of early drug development programs, offering the opportunity of an early and effective integration of pharmacogenetic analyses. Indeed, the use of CYP450 substrates or inhibitors administered together with tamoxifen has been argued to influence treatment outcomes in mammary cancer. Similarly, the response to a drug may be affected by polymorphisms of genes encoding different metabolizing enzymes. Genotyping of the populations included in DDI studies should be recommended when pharmacogenetics is expected to affect pharmacokinetics or pharmacodynamics of any of the active substances. A detailed discussion on evaluation of pharmacogenomic biomarkers in early studies is available in a Guideline edited by EMA [46].

Race may sometime be a proxy for pharmacogenetic differences because toxicities or response-modifying genotypes may occur at different frequencies among races [63]. An example is the warning on the carbamazepine label recommending testing for the $H L A-B{ }^{\star} 1502$ allele in patients of Ham Chinese and Thai ancestry before initiating carbamazepine therapy because of the high risk of developing carbamazepine-induced Stevens-Johnson syndrome and toxic epidermal necrolysis [64]. In general, there are little comparative data available regarding the genetic bases of drug response and toxicity among different racial groups. However, it is important to note that race is not always a good surrogate for pharmacogenetic differences, as evidenced by the fact that $H L A-B^{\star} 5701$ acts as a marker for abacavir hypersensitivity in all races. Thus, further investigation of the basis for marked racial differences in relevant pharmacogenetic pathways, a potential explanation for different response rate and adverse reactions, are worthy of future prospective studies.

Age may also be a variable for pharmacogenetic-driven variability. For example, in the elderly differences in metabolism may be combined with reduced organ function and with the need for multiple medications. The consequences of organ impairment may also be different in genetically different populations and this multiply the variability to drug response. Dosage is certainly crucial in children, where overdosing and underdosing are frequent. Age and weight determine the dosage, but enzymes and transport proteins may be quantitatively and qualitatively different in the paediatric population when compared to adults. Therefore, if a significant impact of a genetic polymorphism on the pharmacokinetic of a drug has been established in adults, the potential consequences in the paediatric and elderly populations should be further elaborated.

\section{Are there methodological issues to be considered?}

Technology advancement has been enormous in the past decade. Indeed, as the cost of whole-genome sequencing is decreasing, the studies to use information even from whole-genome for pharmacogenomic purposes will increase. The first such study was published only last year [65]. In the future, there will probably be an arsenal of new pharmacogenomic markers identified, even in new fiels such as RNA-interference and epigenetic. Therefore, the problem of validation of a marker is still an element to be seriously considered. For a marker whose an acceptable assay is already available, validation should be limited to the disease treatment under consideration. For new pharmacogenomic biomarkers the development of an acceptable assay should parallel the drug development process.

Methodological bias in measurement of a genomic biomarker is an important consideration, mostly for retrospective analyses and for meta-analyses, when different instruments or assays are inevitably used. A centralized measurement facility is always recommended and when this is not possible a careful selection of pooled data sets of predefined criteria could contribute to reduce some types of bias.

Another problem that needs to be regulated from a methodological point of view is the collection of biological samples, often limited by informed consent, patient privacy, intellectual property, and storage issues. Ideally, avenues should be established by which anonymized samples could be collected and stored indefinitely for subsequent analysis, for example in publicly-founded repositories, without risk to the individual patient. Both academic centers and industry could submit and utilize samples as needed for further analysis and the intellectual property that arises from the use of such samples could be shared in public-private partnerships.

\section{What is the impact of adverse event frequency and severity?}

An increasing number of serious adverse reactions have been reported by the FDA between 1998 and 2005. The cost of adverse events is huge and estimated to be $40-50$ billion US\$ per year for the healthcare system. Rare but severe adverse events represent a major reason why drugs are withdrawn after FDA approval. A pharmaceutical company can loose as much as 1 billion US\$ if a marketed drug has been withdrawn. The successful clinical application of pharmacogenomics could increase the likelihood that drugs are effectively dosed and reduce the incidence of adverse reactions associated with drugs. Until recently rare severe allergic reactions to drugs were interpreted as idiosyncratic, but today more and more allergic reactions can be explained by screening for genotypes of the HLA gene locus. However, although it is possible to attempt a replication of a given adverse event with a gene variant [66], it is often difficult to collect sufficient observations of rare adverse drug reactions for genome-wide analysis. 
Certainly, the area of adverse reactions to a given drug is going to remain the most appealing both to the academic investigators and to the industry-driven investigations, but equally important will be the willingness of researchers to reexamine suboptimal pharmacological management programs.

Which are the major questions related to clinical translation of a biomarker?

Despite the growing number of drugs of pharmacogenetic data in the label (www.fda.gov/Drugs/ScienceResearch/ResearchAreas/ Pharmacogenetics/ucm083378.htm), most are not accompanied by recommendations that necessitate immediate genetic testing, although a few labels recommend specific actions to reach a therapeutic decision. The slow pace of the clinical application of pharmacogenetics has several reasons.

Certainly, the first question to be answered for clinical implementation of a pharmacogenomic biomarker is the evidence for its validity and utility, but the consensus on the level of data necessary to establish clinical utility of a given marker is still limited [67]. A gold standard example is offered by studies on abacavir hypersensitivity, where (due to the strength of the evidence base, to the replication in multiple studies, to the confirmation of clinical utility in a specifically designed trial, to the favourable cost-effectiveness data, and to a knowledgeable and vocal patient lobby) both FDA and EMA approved label changes. This culminated in prescribing physicians who are amenable to implement the genomic test request in clinical practice.

However, often even if either the payers would provide reimbursement for routine use of a pharmacogenomic biomarker or the regulatory agencies take increasingly activist stance, it will be necessary to develop easy algorithms to aid physicians in formulating the request of genetic data and in interpreting the results. Education is going to become a strategic effort. Various educational measures will be required, including seminars, symposia, and public assessment reports, as well as the institution of consistent and continuously updated programs at medical and pharmacy schools.

\section{Conclusions}

This meeting was a valuable opportunity to learn and understand the expectations of different stakeholders in the area of pharmacogenetic/ genomic testing within the drug development process. Industry is facing real challenges in generating and interpreting pharmacogenetic/ genomic biomarkers and is supported in this endeavour by the academic centers. The regulatory agencies are promoting development of Reflection Papers and Guidelines that will help the industry to recognize the parameters indicated in the early drug development phases, maintaining frequent communication with the authorities regarding plans and results. The participation of all the stakeholders into this meeting and the preparation of a Reflection Paper by EMA on the topic of designs for pharmacogenetic studies in drug development are important proofs of a common interest towards the development of better medicinal products and an improved practice of medicine.

Pharmacogenetics/genomics has already identified clinically relevant loci which alter the response to several drugs. Such genetic information is now taken into account by drug regulatory agencies, as evidenced by recent drug label modifications integrating pharmacogenetic-based prescription.

However, dramatic efforts need to be made in the selection of genetic tests and in the interpretation of tests for physicians to adapt their treatment on pharmacogenetics. Clinical application will also require a blend of trained clinical workforce, validated genetic tests, payers willing to fund pre-treatment tests, and psychological factors.

Oncology will probably be the most promising field in pharmacogenomics, because the tumoral genomic variability is enormous, new targeted anticancer drugs are several, and new selective tests might really bring benefits to patients.

Examples of constitutional or somatic pharmacogenetic tests that are clinically available and relevant to cancer drug selection or dosing or both are: $U G T 1 A 1^{*} 28$ gene variant and neutropenia in irinotecan therapy for colon cancer [68]; CYP2D6 gene polymorphisms and clinical efficacy of tamoxifen in estrogen receptor-positive breast cancers $(\mathrm{H})$; and PTPMT gene variants and mercaptopurine use in lymphoid malignancies [69].

In conclusion, the development of new effective pharmacogenetic biomarkers in drug development is the common goal for regulatory authorities, academia, and industry. To achieve this, it is important that regulatory requirements do not hinder innovation and vice versa. A dialogue is ongoing among the different stakeholders, whose responsibility is to ensure continuous exchange in an environment characterized by rapid scientific advances and global development programs. This integration is already contributing to the improvement in the current health scenario.

\section{Highlights from the meeting}

The intention of this paper was to provide an evidence based considerations of genomic biomarkers related to drug development, with focus on patient selection and trial methodology.

The main recommendations derived from this work were recognized in:

- Predictive genomic biomarkers should occupy the highest area of interest

- The selection and evaluation of the genomic biomarkers in any development programme should be dependent on the expected primary role on the genomic biomarker under consideration

- The patient selection would be enhanced using a genomic biomarker for better definition of disease prognosis, for excluding patients at increased risk and for prediction of drug response

- Using of a central laboratory may reduce the risk of different misclassifications

- Validation studies can be prospective or retrospective, nonrandomized or randomized

- Irrespective of the type of trials used for exploration, it is important to collect information on the performance of the biomarkers for predictability and clinical validity

- For newly identified or specific genomic biomarkers, development of a specific assay/KIT might be necessary

\section{Acknowledgements}

This work was supported by an unrestricted grant of the Group for the Respect of Ethics and Excellence in Science (GREES). 
Citation: Brandi ML, Eric A, Ann D, Willard D, Dominique E, et al. (2012) Challenges Faced in the Integration of Pharmacogenetics/Genomics into Drug Development. J Pharmacogenom Pharmacoproteomics 3:108. doi:10.4172/2153-0645.1000108

\section{References}

1. Beutler E (1969) Drug-induced hemolytic anemia. Pharmacol Rev 21: 73-103.

2. Wang L, McLeod HL, Weinshilboum RM (2011) Genomics and drug response. N Engl J Med 364: 1144-1153.

3. Lister R, Pelizzola M, Dowen RH, Hawkins RD, Hon G, et al. (2009) Human DNA methylomes at base resolution show widespread epigenomic differences. Nature 462: 315-322.

4. Passetti F, Ferreira CG, Costa FF (2009) The impact of microRNAs and alternative splicing in pharmacogenomics. Pharmacogenomics J 9: 1-13.

5. Sadee W, Wang D, Papp AC, Pinsonneault JK, Smith RM, et al. (2011) Pharmacogenomics of the RNA world: structural RNA polymorphisms in drug therapy. Clin Pharmacol Ther 89: 355-365.

6. Shin J, Kayser SR, Langaee TY (2009) Pharmacogenetics: from discovery to patient care. Am J Health Syst Pharm 66: 625-637.

7. Frueh FW, Amur S, Mummaneni P, Epstein RS, Aubert RE, et al. (2008) Pharmacogenomic biomarker information in drug labels approved by the United States food and drug administration: prevalence of related drug use. Pharmacotherapy 28: 992-998.

8. Feero WG, Guttmacher AE, Collins FS (2010) Genomic medicine--an updated primer. N Engl J Med 362: 2001-2011.

9. Wang L, McLeod HL, Weinshilboum RM (2011) Genomics and drug response. N Engl J Med 364: 1144-1153.

10. US Food and Drug Administration. Innovation or stagnation: challenge and opportunity of the clinical path to new medical product.

11. Keatley KL (1999) A review of US EPA and FDA requirements for electronic records, electronic signatures, and electronic submissions. Qual Assur 7: 7789.

12. US Food and Drug Administration (2008) E15 Definitions for Genomic Biomarkers, Pharmacogenomics, Pharmacogenetics, Genomic Data and Sample Coding Categories: Guidance for Industry. Fed Regist 73: 19074.

13. European Medicines Agency (2002) Position paper on terminology on Pharmacogenetics. EMEA/CPMP/3070/01

14. European Medicines Agency (2007) Reflection paper on pharmacogenomic samples, testing and data handling. EMEA/CHMP/201914.

15. Food and Drug Administration, HHS (2008) International Conference on Harmonisation; Guidance on E15 Pharmacogenomics Definitions and Sample Coding; Availability. Notice. Fed Regist 73: 19074-19076.

16. European Medicines Agency (2007) Reflection paper on the use of pharmacogenetics in the pharmacokinetic evaluation of medicinal products. EMEA/128517/2006

17. Woodcock J (2010) Assessing the clinical utility of diagnostics used in drug therapy. Clin Pharmacol Ther 88: 765-773.

18. Bromley CM, Close S, Cohen N, Favis R, Fijal B, et al. (2009) Designing pharmacogenetic projects in industry: practical design perspectives from the Industry Pharmacogenomics Working Group. Pharmacogenomics J 9: 14-22.

19. Roses $A D$ (2008) Pharmacogenetics in drug discovery and development: a translational perspective. Nat Rev Drug Discov 7: 807-817.

20. Ingelman-Sundberg M (2008) Pharmacogenomic biomarkers for prediction of severe adverse drug reactions. N Engl J Med 358: 637-639.

21. Jorgensen AL, Williamson PR (2008) Methodological quality of pharmacogenetic studies: issues of concern. Stat Med 27: 6547-6569.

22. Council for International Organizations of Medical Sciences (CIOMS) (2005) Pharmacogenetics: towards improving treatments with medicines, Geneva, Switzerland.

23. Nuffield Council on Bioethics (2003) Pharmacogenetics: ethical issues.

24. Stingl Kirchheiner JC, Brockmöller J (2011) Why, when, and how should pharmacogenetics be applied in clinical studies?: current and future approaches to study designs. Clin Pharmacol Ther 89: 198-209.

25. Kwon JM, Goate AM (2000) The candidate gene approach. Alcohol Res Health 24: 164-168.
26. Rettie AE, Wienkers LC, Gonzalez FJ, Trager WF, Korzekwa KR (1994) Impaired (S)-warfarin metabolism catalysed by the R144C allelic variant of CYP2C9. Pharmacogenetics 4: 39-42.

27. Steward DJ, Haining RL, Henne KR, Davis G, Rushmore TH, et al. (1997) Genetic association between sensitivity to warfarin and expression of CYP2C9*3. Pharmacogenetics 7: 361-367.

28. Veenstra DL, Blough DK, Higashi MK, Farin FM, Srinouanprachan S, et al. (2005) CYP2C9 haplotype structure in European American warfarin patients and association with clinical outcomes. Clin Pharmacol Ther 77: 353-364.

29. Hirschhorn JN, Daly MJ (2005) Genome-wide association studies for common diseases and complex traits. Nat Rev Genet 6: 95-108.

30. Rieder MJ, Reiner AP, Gage BF, Nickerson DA, Eby CS, et al. (2005) Effect of VKORC1 haplotypes on transcriptional regulation and warfarin dose. $\mathrm{N}$ Engl Med 352: 2285-2293.

31. Kathiresan S, Newton-Cheh C, Gerszten RE (2004) On the interpretation of genetic association studies. Eur Heart J 25: 1378-1381.

32. Wang WY, Barratt BJ, Clayton DG, Todd JA (2005) Genome-wide association studies: theoretical and practical concerns. Nat Rev Genet 6: 109-118.

33. Kindmark A, Jawaid A, Harbron CG, Barratt BJ, Bengtsson OF, et al. (2008) Genome-wide pharmacogenetic investigation of a hepatic adverse even without clinical signs of immunopathology suggests an underlying immune pathogenesis. Pharmacogenomics J 8: 186-195.

34. Schütz E, Gummert J, Mohr FW, Armstrong VW, Oellerich M (1996) Should 6 -thioguanine nucleotides be monitored in heart transplant recipients given azathioprine? Ther Drug Monit 18: 228-233.

35. Mulder H, Heerdink ER, van lersel EE, Wilmink FW, Egberts AC (2007) Prevalence of patients using drugs metabolized by cytochrome P450 2D6 in different populations: a cross-sectional study. Ann Pharmacother 41: 408-413.

36. Sackett DL (1979) Bias in analytic research. J Chronic Dis 32: 51-63.

37. Weiss ST, Silverman EK, Palmer LJ (2001) Case-control association studies in pharmacogenetics. Pharmacogenomics J 1: 157-158.

38. Lin E, Chen PS (2008) Pharmacogenomics with antidepressants in the STAR*D study. Pharmacogenomics 9: 935-946.

39. SEARCH Collaborative Group, Link E, Parish S, Armitage J, Bowman L, et al. (2008) SLCO1B1 variants and statin-induced myopathy--a genomewide study. N Engl J Med 359: 789-799.

40. Kalow W, Tang BK, Endrenyi L (1998) Hypothesis: comparisons of inter- and intra-individual variations can substitute for twin studies in drug research Pharmacogenetics 8: 283-289.

41. Hulley SB, Cummings SR, Browner WS, Grady DG, Newman TB (2001) Designing Clinical Research. Lippincott, Williams \& Wilkins, Philadelphia, PA USA.

42. Soumerai SB, Ross-Degnan D, Avorn J, McLaughlin T, Choodnovskiy I (1991) Effects of Medicaid drug-payment limits on admission to hospitals and nursing homes. N Engl J Med 325: 1072-1077.

43. Kelly PJ, Stallard N, Whittaker JC (2005) Statistical design and analysis of pharmacogenetic trials. Stat Med 24: 1495-1508.

44. Uhr M, Tontsch A, Namendorf C, Ripke S, Lucae S, et al. (2008) Polymorphisms in the drug transporter gene $\mathrm{ABCB} 1$ predict antidepressant treatment response in depression. Neuron 57: 203-209.

45. Food and Drug Administration, HHS (2011) International Conference on Harmonisation; Guidance on E16 Biomarkers Related to Drug or Biotechnology Product Development: Context, Structure, and Format of Qualification Submissions; availability. Notice. Fed Regist 76: 49773-49774.

46. Guideline on the use of pharmacogenetic methodologies in the pharmacokinetic evaluation of medicinal products A/CHMP/37646/2009.

47. Sim SC, Ingelman-Sundberg M (2011) Pharmacogenomic biomarkers: new tools in current and future drug therapy. Trends Pharmacol Sci 32: 72-81.

48. Frueh FW, Amur S, Mummaneni P, Epstein RS, Aubert RE, et al. (2008) Pharmacogenomic biomarker information in drug labels approved by the United States food and drug administration: prevalence of related drug use. Pharmacotherapy 28: 992-998. 
Citation: Brandi ML, Eric A, Ann D, Willard D, Dominique E, et al. (2012) Challenges Faced in the Integration of Pharmacogenetics/Genomics into Drug Development. J Pharmacogenom Pharmacoproteomics 3:108. doi:10.4172/2153-0645.1000108

Page 10 of 10

49. Relling MV, Altman RB, Goetz MP, Evans WE (2010) Clinical implementation of pharmacogenomics: overcoming genetic exceptionalism. Lancet Oncol 11: 507-509.

50. Swen JJ, Nijenhuis M, de Boer A, Grandia L, Maitland-van der Zee AH, et al. (2011) Pharmacogenetics: from bench to byte--an update of guidelines. Clin Pharmacol Ther 89: 662-673.

51. Landon MR (2005) Ethics and policy perspectives on personalized medicine in the post-genomic era. J Biolaw Bus 8: 28-36.

52. Nies AT, Schwab M, Keppler D (2008) Interplay of conjugating enzymes with OATP uptake transporters and ABCC/MRP efflux pumps in the elimination of drugs. Expert Opin Drug Metab Toxicol 4: 545-568.

53. Niemi M (2010) Transporter pharmacogenetics and statin toxicity. Clin Pharmacol Ther 87: 130-133.

54. Schwarz UI, Stein CM (2006) Genetic determinants of dose and clinica outcomes in patients receiving oral anticoagulants. Clin Pharmacol Ther 80 $7-12$.

55. Liu LW, Liu H, Chen GL, Huang YL, Han LL, et al. (2010) Angiotensinconverting enzyme gene I/D genotype affected metoprolol-induced reduction in 24-hour average heart rate. Chin Med J (Engl) 123: 1382-1386.

56. Dishy V, Sofowora GG, Xie HG, Kim RB, Byrne DW, et al. (2001) The effect of common polymorphisms of the beta2-adrenergic receptor on agonist-mediated vascular desensitization. N Engl J Med 345: 1030-1035.

57. Lynch TJ, Bell DW, Sordella R, Gurubhagavatula S, Okimoto RA, et al. (2004) Activating mutations in the epidermal growth factor receptor underlying responsiveness of non-small-cell lung cancer to gefitinib. N Engl J Med 350: 2129-2139.

58. Druker BJ (2008) Translation of the Philadelphia chromosome into therapy for CML. Blood 112: 4808-4817.

59. Samson M, Libert F, Doranz BJ, Rucker J, Liesnard C, et al. (1996) Resistance to HIV-1 infection in caucasian individuals bearing mutant alleles of the CCR-5 chemokine receptor gene. Nature 382: 722-725.

60. Chasman DI, Posada D, Subrahmanyan L, Cook NR, Stanton VP, et al. (2004)
Pharmacogenetic study of statin therapy and cholesterol reduction. JAMA 291: 2821-2827.

61. Biomarker qualification-renal biomarkers.

62. Engen RM, Marsh S, Van Booven DJ, McLeod HL (2006) Ethnic differences in pharmacogenetically relevant genes. Curr Drug Targets 7: 1641-1648.

63. US Food and Drug Administration. Information for healthcare professionals Dangerous or Even Fatal Skin Reaction - carbamazepine (marketed as Carbatrol, Equetro, Tegretol, and generics).

64. Ashley EA, Butte AJ, Wheeler MT, Chen R, Klein TE, et al. (2010) Clinical assessment incorporating a personal genome. Lancet 375: 1525-1535.

65. Daly AK, Donaldson PT, Bhatnagar P, Shen Y, Pe'er I, et al. (2009) HLA-B*5701 genotype is a major determinant of drug-induced liver injury due to flucloxacillin Nat Genet 41: 816-819.

66. Woodcock $\mathrm{J}$ (2010) Assessing the clinical utility of diagnostics used in drug therapy. Clin Pharmacol Ther 88: 765-773.

67. Mallal S, Nolan D, Witt C, Masel G, Martin AM, et al. (2002) Association between presence of HLA-B*5701, HLA-DR7, and HLA-DQ3 and hypersensitivity to HIV-1 reverse-transcriptase inhibitor abacavir. Lancet 359: 727-732.

68. de Jong FA, de Jonge MJ, Verweij J, Mathijssen RH (2006) Role of pharmacogenetics in irinotecan therapy. Cancer Lett 234: 90-106.

69. Stocco G, Crews KR, Evans WE (2010) Genetic polymorphism of inosinetriphosphate-pyrophosphatase influences mercaptopurine metabolism and toxicity during treatment of acute lymphoblastic leukemia individualized for thiopurine-S-methyl-transferase status. Expert Opin Drug Saf 9: 23-37.

70. Ioannidis JP (2006) Commentary: grading the credibility of molecular evidence for complex diseases. Int J Epidemiol 35: 572-578.

71. Ioannidis JP, Boffetta P, Little J, O'Brien TR, Uitterlinden AG, et al. (2008) Assessment of cumulative evidence on genetic associations: interim guidelines. Int J Epidemiol 37: 120-132.

72. Goetz MP, Rae JM, Suman VJ, Safgren SL, Ames MM, et al. (2005) Pharmacogenetics of tamoxifen biotransformation is associated with clinical outcomes of efficacy and hot flashes. J Clin Oncol 23: 9312-9318. 